\title{
Caracterización y análisis y de un recubrimiento multicapa con DLC como capa final
}

\section{Characterization and analysis of a multilayer coating with DLC as top layer}

Presentación: 6-7/10/2020

\section{Doctorando:}

\section{Francisco A. Delfin}

Grupo de Ingeniería de Superficies (GIS), Facultad Regional Concepción del Uruguay, Universidad Tecnológica Nacional - Argentina delfinf@frcu.utn.edu.ar

\section{Directora:}

\section{Sonia P. Brühl}

Co-director:

\section{Ricardo C. Dommarco}

\section{Resumen}

En este trabajo se estudia un recubrimiento multicapa DLC producido por primera vez en la industria nacional, utilizando un equipo PVD PEMS. La base es una capa de anclaje de AlTiN y la capa exterior es carbono amorfo hidrogenado dopado con cromo (DLC), que le confiere excelentes propiedades tribológicas, como un muy bajo coeficiente de fricción y una buena resistencia al desgaste. Se utiliza la nitruración iónica como pretratamiento, lo que mejora notablemente la adhesión al substrato, pero no incide en la resistencia al desgaste por contacto deslizante en las condiciones que fueron ensayadas.

Palabras clave: DLC, PVD, PEMS, recubrimientos, dúplex, desgaste, industria nacional

\begin{abstract}
In this work a DLC multilayer coating produced for the first time in Argentina, is analyzed. It is deposited using PVD PEMS. The first layer is made of AITiN and the outer layer is a chrome-doped hydrogenated amorphous carbon (DLC), which gives it an excellent tribological behavior, with a very low friction coefficient and good wear resistance. Ion nitriding is used as a pretreatment, which significantly improves adhesion to the substrate, but makes no difference on the resistance to sliding contact wear with the used testing parameters.
\end{abstract}

Keywords: DLC, PVD, PEMS, coatings, duplex, wear

\section{Introducción}

La industria de las herramientas de corte y los insertos emplean recubrimientos duros para lograr mayor duración y vida útil del filo. La extensión a otras áreas industriales que requieran un control de la fricción, el desgaste y la corrosión, está aún en desarrollo, pero representa un gran interés por parte de varios actores del medio que buscan reducir costos operativos y de mantenimiento. Para lograr estas películas protectoras, se suele recurrir a técnicas PVD, como la deposición por arco o el sputtering, siendo este último el que produce mejor acabado superficial, libre de macropartículas, aunque a una velocidad mucho más lenta. Para solucionar esto, se ha desarrollado el proceso de magnetrón sputtering asistido por plasma, o PVD PEMS (Keunecke et al., 2010). Con esta técnica es posible depositar películas finas, e incluso múltiples capas de diferentes materiales, para combinar distintas propiedades. Un ejemplo de este tipo de recubrimiento es el AlTiN, que posee alta dureza superficial y es 
usado en herramientas de corte (Bouzakis et al., 2012). Sin embargo, su coeficiente de fricción no es muy bajo (Erkens et al., 2004). Otros recubrimientos, muy utilizados por su bajo coeficiente de fricción y alta dureza superficial, son los de base carbono tipo DLC, que además resisten muy bien a la corrosión (Dalibon et al., 2015).

Cuando se deposita este tipo de recubrimientos sobre aceros de baja aleación, que son más blandos que los aceros para herramientas, la adhesión suele verse muy comprometida. Para utilizar estas películas sobre elementos de máquinas, manteniendo un núcleo tenaz pero un exterior duro y con bajo coeficiente de fricción, es necesario utilizar como tratamiento previo otros métodos de tratamiento superficial, como es el caso de la nitruración iónica, que es un proceso de endurecimiento por difusión. La nitruración iónica sobre aceros como el AISI 4140 ya está muy desarrollada (Corengia et al., 2005), e incluso está probado que el gradiente de dureza generado en el sustrato ayuda a mejorar notablemente la adhesión de recubrimientos duros sobre este tipo de aceros (Forsich et al., 2014). La combinación de dos tipos de tratamientos diferentes se conoce con el nombre de proceso dúplex.

En este trabajo se presenta la caracterización de un recubrimiento multicapa, formado por la combinación de varias capas finas diferentes hechas secuencialmente en el mismo equipo, que son producidos por primera vez en el país. Se estudia la influencia del pretratamiento de nitruración iónica sobre la adhesión y la resistencia al desgaste. Esta investigación se encuentra en el marco de un proyecto de colaboración entre Coating.Tech (Tantal SRL), IONAR S.A., YPF Tecnología S.A. y el Grupo de Ingeniería de Superficies (UTN FRCU).

\section{Desarrollo}

Se estudiaron muestras de acero de baja aleación AISI 4140 (ver composición obtenida mediante GDOES en la Tabla 1). Las mismas fueron cortadas de una barra bonificada de sección circular de $60 \mathrm{~mm}$ de diámetro y aproximadamente $6 \mathrm{~mm}$ de altura. La superficie a recubrir fue desbastada con papel de lija al agua de granulometría creciente y finalmente pulida con pasta de diamante de 1,5 $\mu \mathrm{m}$. Un grupo de muestras fue enviada a la empresa IONAR S.A. (San Martín, Buenos Aires), donde se llevó a cabo la nitruración iónica a $500{ }^{\circ} \mathrm{C}$ por 25 horas. Se midió la dureza en superficie mediante micro-indentación Vickers con carga $50 \mathrm{~g}\left(\mathrm{HV}_{0,05}\right)$. Se cortó una probeta y se incluyó en resina termoformable para ver la capa de compuesto en microscopio óptico y se midió el perfil de dureza en profundidad luego de nitrurada con carga de $25 \mathrm{~g}\left(\mathrm{HV}_{0,025)}\right.$.

\begin{tabular}{|c|c|c|c|c|c|c|c|c|}
\hline $\mathbf{C} \%$ & $\mathbf{S i} \%$ & $\mathbf{M n} \%$ & $\mathbf{C r} \%$ & $\mathbf{N i} \%$ & $\mathbf{M o \%}$ & $\mathbf{S \%}$ & $\mathbf{P \%}$ & $\mathbf{C u} \%$ \\
\hline 0,419 & 0,273 & 0,850 & 0,976 & 0,153 & 0,188 & $<0,001$ & 0,009 & 0,086 \\
\hline
\end{tabular}

Tabla 1: Composición real del acero AISI 4140 utilizado.

Luego se enviaron muestras nitruradas y no nitruradas para recubrir con una película multicapa en un equipo comercial de la marca CemeCon AG, propiedad de la empresa Coating.Tech de Tantal SRL (Santa Rosa de Calamuchita, Córdoba). Esta película consiste en una capa de anclaje de AlTiN con un recubrimiento superior fino de carbono amorfo hidrogenado (DLC) dopado con cromo, utilizando capas intermedias de transición. El equipo de deposición es un PVD PEMS reactivo, donde se utilizan dos targets o blancos de titanio y de aluminio para la primera etapa utilizando nitrógeno como gas reactivo. Para hacer las capas múltiples y los gradientes, los blancos de $\mathrm{Al}$ y Ti se van retirando progresivamente, mientras se introducen otros dos blancos, de cromo y de grafito. En este caso, también cambia el gas reactivo, ya que se merma el caudal de nitrógeno y se introduce poco a poco acetileno. Por lo tanto, disminuye paulatinamente la formación de nitruros de titanio y aluminio para dar lugar a los nitruros de cromo $\mathrm{CrN}$, carbonitruros de cromo $\mathrm{CrCN}$ y carburos de cromo $\mathrm{CrC}$, para finalmente terminar con la capa exterior de carbono amorfo dopado con cromo, que será la responsable de las propiedades tribológicas de este recubrimiento.

Para determinar el espesor del recubrimiento, se utilizó el ensayo de calotest. A pesar de su conocida fiabilidad y sencillez, este método no permite determinar correctamente el espesor de las múltiples capas, sobre todo cuando algunas pueden tener un espesor del orden de los $500 \mathrm{~nm}$. Por ello, también se seccionaron las muestras y se realizó un tratamiento de pulido iónico sobre la sección transversal, para medir el espesor del recubrimiento con microscopio electrónico (SEM), donde además se realizaron mediciones de composición química por medio de la técnica EDS. En una muestra solo recubierta, se hizo Difracción de Rayos X de ángulo rasante con ánodo de cobre, para determinar las fases cristalinas presentes en el recubrimiento.

A fin de evaluar las propiedades tribológicas del recubrimiento, se utilizó una máquina de ensayo de desgaste por deslizamiento tipo Pin-On-Disk de construcción propia, que responde a la norma ASTM G99, siendo el disco la muestra a ensayar y utilizando una bolilla de alúmina de $6 \mathrm{~mm}$ de diámetro como contraparte, con recorridos de 
$500 \mathrm{~m}$ y $1000 \mathrm{~m}$ y cargas de $10 \mathrm{~N}$ y $12 \mathrm{~N}$, respectivamente. El coeficiente de fricción se registró en tiempo real durante todo el ensayo, la huella de desgaste se analizó con un perfilómetro mecánico y se observó al microscopio óptico. Los ensayos de desgaste se realizaron a temperatura ambiente promedio de $(16.4 \pm 0.8){ }^{\circ} \mathrm{C}$ y la humedad relativa variable entre $39 \%$ y $62 \%$. Con el objeto de ensayar la resistencia a la adhesión de los recubrimientos se hicieron ensayos de Scratch Test de carga constante, variando cargas en cada ensayo entre $5 \mathrm{~N}$ y $70 \mathrm{~N}$, utilizando un perfilómetro mecánico y un microscopio óptico para evaluar los resultados. También se realizaron ensayos de indentación Rockwell C para evaluar la tenacidad a la fractura del recubrimiento. Si bien el ensayo de indentación es utilizado en numerosos trabajos para medir la adhesión, según la propia norma VDI 3198 (Vidakis et al., 2003), no es un método recomendable para recubrimientos con un espesor mayor a $3 \mu \mathrm{m}$, aunque igualmente aporta información valiosa sobre el comportamiento del conjunto ante la aplicación de una carga normal estática (150 kg).

\section{Resultados}

Como parte del control de calidad previo al inicio de los tratamientos y ensayos, se midió la dureza del material adquirido, el acero AISI 4140 bonificado, que resultó en promedio $(320 \pm 10) \mathrm{HV}$. Esto permite estimar que la temperatura de revenido fue alrededor de $550{ }^{\circ} \mathrm{C}$, lo cual es conveniente dado que la nitruración se realizó a una temperatura algo menor.

Las muestras nitruradas presentaron una dureza mayor, en promedio $(840 \pm 42) \mathrm{HV}$ en su superficie. Se realizó también un perfil de dureza en profundidad, donde se observó que la penetración del tratamiento fue de $300 \mu \mathrm{m}$.

En las muestras recubiertas, primero se hicieron mediciones con calotest, donde se determinó que la película tiene un espesor promedio total de $(9,8 \pm 0,8) \mu \mathrm{m}$. Utilizando microscopía electrónica de barrido, se observó que consiste en 6 capas, como se aprecia en la Figura 1a, más un interlayer o capa de adherencia. Con la técnica de difracción de Rayos X (Figura 1b) y el análisis de EDS en el SEM, se determinó la composición química y la estructura de cada capa.

La deposición del interlayer o intercapa, que fue lograda mediante el bombardeo de iones de Ti puro contra el substrato y tiene un espesor de entre 50 y $100 \mathrm{~nm}$. Sobre esta capa delgada, se depositó el principal componente, que es el AlTiN, con un espesor de unos $5 \mu \mathrm{m}$, que funciona como anclaje al substrato y brinda resistencia mecánica. Luego siguen varias capas finas alternadas de entre 200 y $800 \mathrm{~nm}$ hasta llegar a la superficie, donde se depositó el "DLC", que se detecta claramente en la Figura 1 de color gris más oscuro y resultó ser carbono amorfo dopado con cromo. Las capas intermedias actúan como un gradiente de dureza y composición entre el AlTiN y el DLC para favorecer la adherencia. Estas capas intermedias están hechas de Ti y un gradiente de nitruros, carbonitruros y carburos de cromo, que finalmente termina mezclándose con el DLC, la capa superior que interactúa con el medio y será la responsable del comportamiento tribológico.
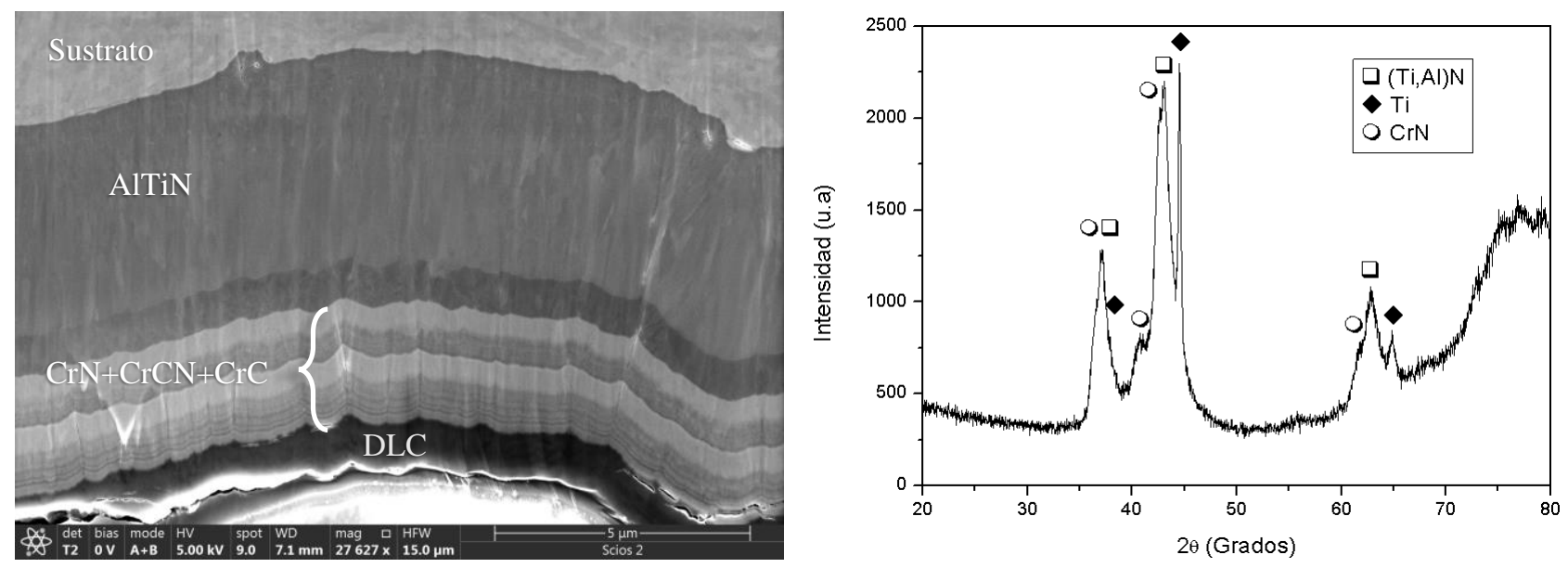

Figura 1: a) Micrografía SEM de la sección transversal del recubrimiento; b) Difracción por Rayos X.

Analizando particularmente el difractograma de la Figura $1 \mathrm{~b}$, se puede observar la presencia de los picos de titanio, nitruro de titanio aluminio, como así también de $\mathrm{CrN}$, que son similares para todo el conjunto $\mathrm{CrN}-\mathrm{CrCN}-\mathrm{CrC}$. La parte del difractograma donde aumenta el fondo, que comienza a partir de los $50^{\circ}$ y se hace más pronunciado a partir de los $65^{\circ}$, responde a la presencia de una estructura amorfa en la superficie del recubrimiento, correspondiente a la película de carbono amorfo hidrogenado, donde el hidrógeno proviene del acetileno y es el que impide la formación de una estructura cristalina. Este tipo de estructura tiene usualmente uniones del tipo C-H y C-C 
(grafítica), lo que se traduce en un buen comportamiento tribológico, con un muy bajo coeficiente de fricción, lo que contribuye a disminuir el desgaste.

En los ensayos tribológicos utilizando una carga de $10 \mathrm{~N}$ y una distancia recorrida de $500 \mathrm{~m}$, se reveló una gran diferencia entre el desempeño de la muestra nitrurada y las muestras recubiertas, ya sea dúplex o sólo con el recubrimiento. En la Figura 2a se observa la evolución del coeficiente de fricción $(\mu)$ con el tiempo, siendo el resultado en estado estable para la muestra nitrurada $\mu \sim 0.65$, y para las muestras recubiertas $\mu \sim 0.2$, es decir, una reducción del $\sim 70 \%$ del coeficiente de fricción.

Cuando se analizó el volumen de desgaste sufrido por las muestras, la nitrurada presentó una pérdida de volumen $\sim 9$ veces mayor que las recubiertas, las que tuvieron aproximadamente el mismo rendimiento.
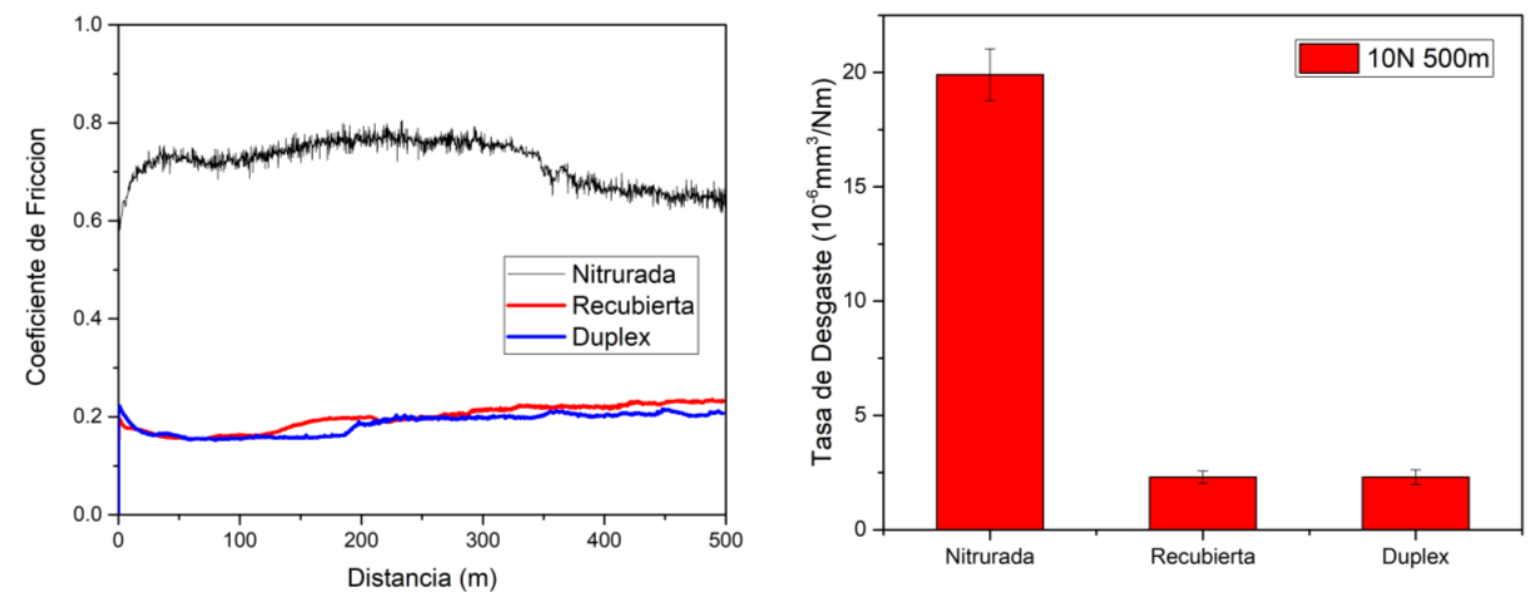

Figura 2: a) Coeficiente de fricción; b) Tasa de desgaste; para ensayos con carga de $10 \mathrm{~N}$ y longitud $500 \mathrm{~m}$.

Para intentar encontrar una diferencia entre las muestras dúplex y solo recubierta, se las sometió a una condición de ensayo más severa, utilizando una carga de $12 \mathrm{~N}$ y una distancia deslizante de $1000 \mathrm{~m}$. En la Figura 3 se muestran los resultados de fricción y desgaste, observándose un incremento en la fricción $\mu \sim 0.35$ para ambas muestras, a causa del aumento de la superficie real de contacto debido a una carga mayor. La tasa de desgaste de las muestras dúplex y recubierta resultó otra vez muy similar, aunque en este caso un $\sim 50 \%$ de la tasa de desgaste medida en el ensayo a menor carga.
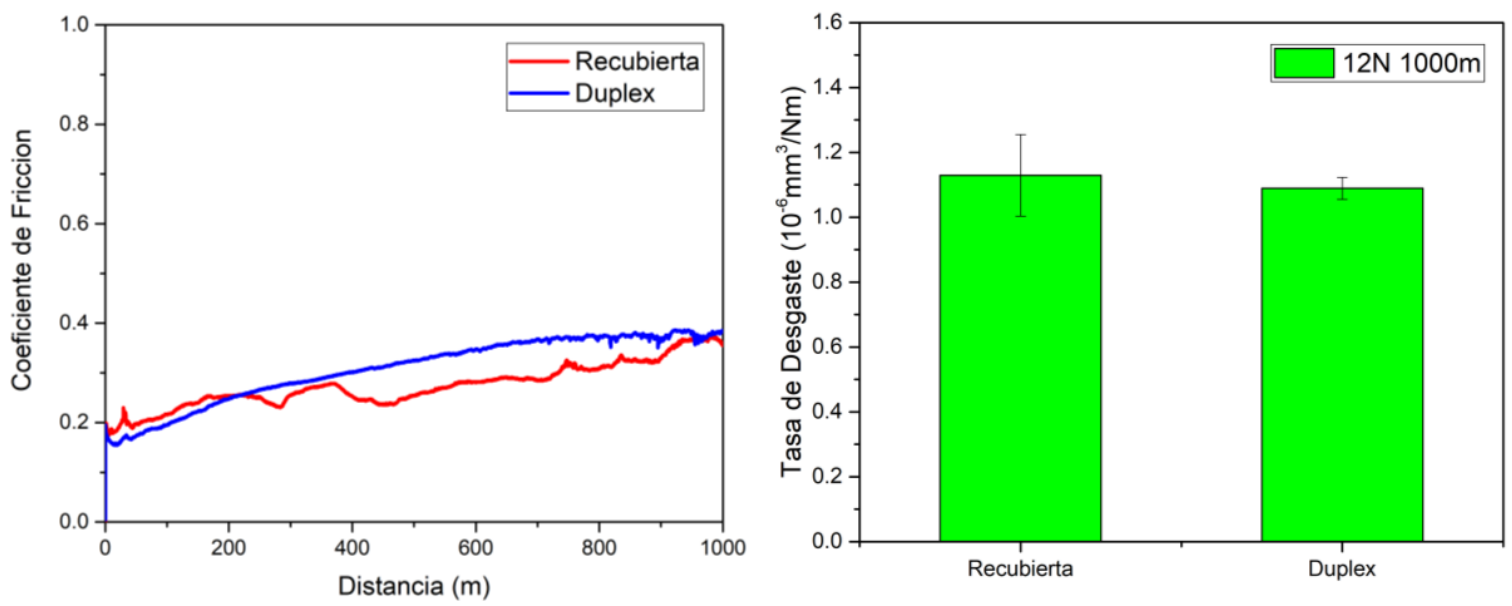

Figura 3: a) Coeficiente de fricción; b) Tasa de desgaste; para carga de 12 N y longitud 1000 m.

En los ensayos de rayado o Scratch Test, analizando con microscopio óptico la huella para una carga de $50 \mathrm{~N}$, se puede determinar que la muestra recubierta presentó una delaminación completa, mientras que la muestra dúplex, con la misma carga, apenas comienza a presentar escamado, como se ve en la Figura 4. La delaminación completa de la muestra dúplex se obtuvo para una carga de $90 \mathrm{~N}$, lo que representa una mejora del $80 \%$ en la carga crítica. Usando el perfilómetro en las huellas de Scratch de $50 \mathrm{~N}$, se midió una profundidad de $16 \mu \mathrm{m}$ en la probeta recubierta, mientras que en la probeta dúplex resultó $6 \mu \mathrm{m}$, lo que indica que el rayado no llegó a penetrar completamente el espesor de la película. Este comportamiento es el esperado, ya que la capa nitrurada ofrece una mayor capacidad de carga que el sustrato de acero sin modificar. Esto quedó en evidencia en este tipo de ensayos, 
donde la mayor carga normal, el menor diámetro de la punta de rayado y los esfuerzos tangenciales son muy superiores a los que se presentan en el ensayo Pin-on-Disk.
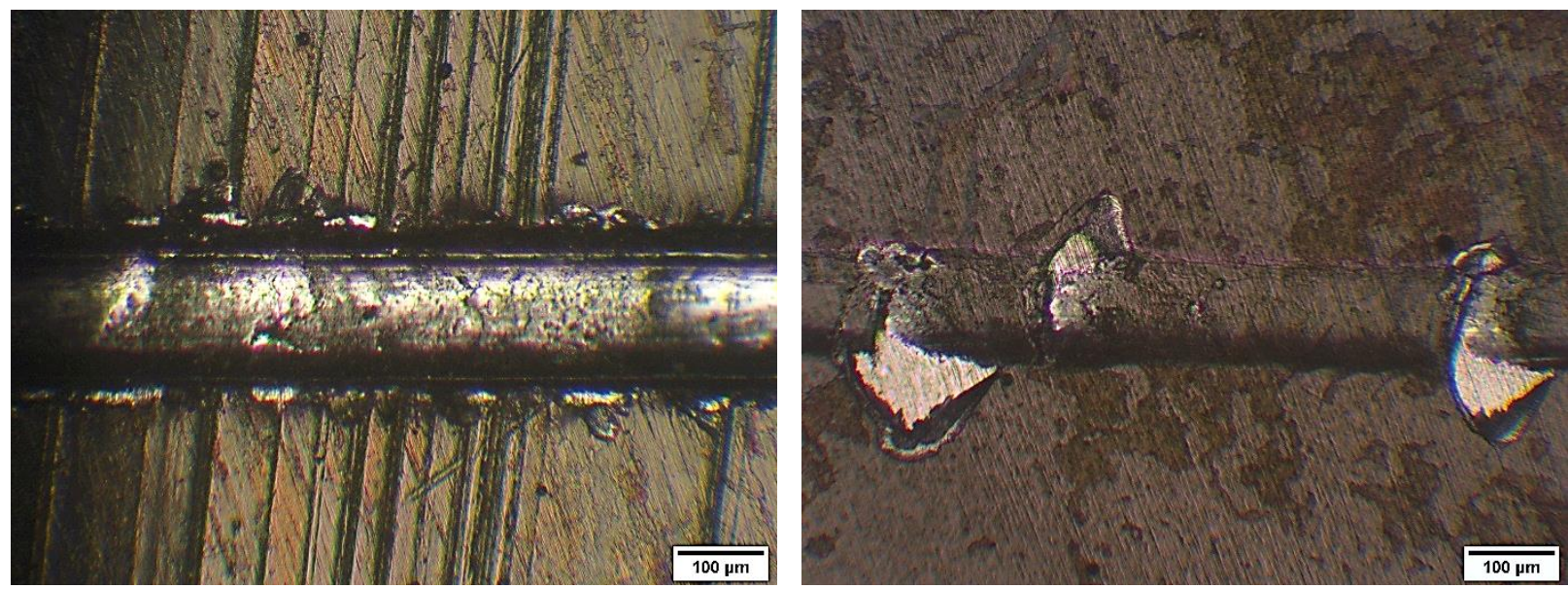

Figura 4: Scratch test $50 \mathrm{~N}$, muestra solo recubierta (a) y muestra dúplex (b).

El análisis de los ensayos de indentación Rockwell C en el microscopio óptico, mostró una clara diferencia entre las muestras, como se ve en la Figura 5. En la probeta solo recubierta, se ven fracturas de la película en los alrededores de la indentación y mucha deformación plástica, que resulta en la formación de una protuberancia en el borde de la indentación. En la muestra dúplex en cambio, no se observó fractura del recubrimiento y la deformación plástica es moderada y prácticamente imperceptible en el microscopio óptico, por lo que se puede afirmar que su tenacidad a la fractura es mayor. Esto se debe a un gradiente de dureza favorable que aporta la capa nitrurada con su capa de compuestos y zona de difusión, que provee una mayor capacidad de carga.
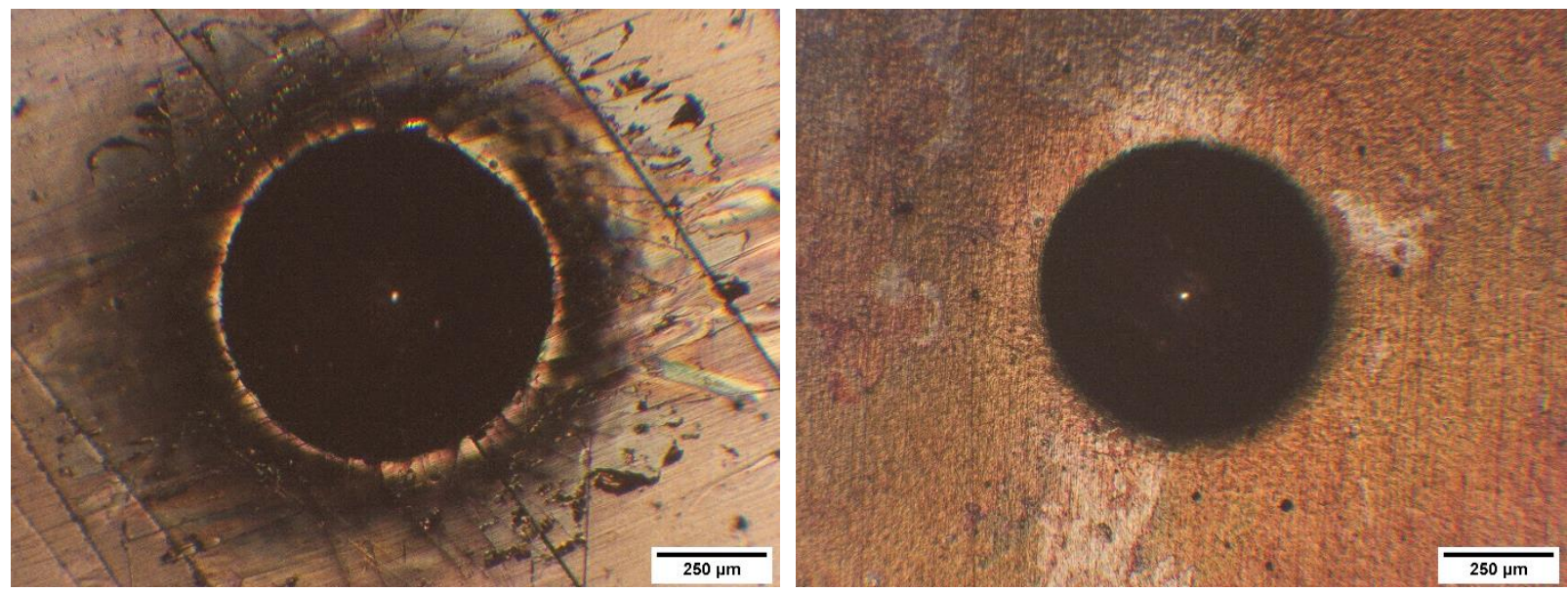

Figura 5: Indentación Rockwell C, muestra solo recubierta (a) y muestra dúplex (b).

En este punto, cabe destacar que tanto los resultados de Scratch Test como los de indentación Rockwell C son mejores que los obtenidos en otros recubrimientos crecidos por PVD por arco, como por ejemplo el TiN, y que fueron analizados previamente por este Grupo de Investigación. Por lo tanto, se puede determinar que se trata de un recubrimiento que tiene un muy buen comportamiento frente a la fricción y al desgaste, que incluso puede ser mejorado con un pretratamiento, como la nitruración iónica del acero utilizada en este caso.

\section{Conclusiones}

Este recubrimiento multicapa resultó muy efectivo para la resistencia al desgaste por deslizamiento, aun cuando se utilizaron cargas altas. Como ya se ha mencionado, esto se debe a las características propias de los recubrimientos DLC hidrogenados, que presentan un comportamiento grafítico. Incluso se pudo determinar que, para el escenario tribológico estudiado, no existe mucha incidencia del pretratamiento, ya que la solicitación no llega a la interface o al 
substrato. El hecho de que el coeficiente de fricción sea muy bajo es determinante, ya que las fuerzas tangenciales son más bajas que para el caso de recubrimientos con mayor coeficiente de fricción.

Los ensayos de Scratch Test revelaron que las muestras dúplex tienen una carga critica mayor, por lo que se puede afirmar que la adherencia mejora con el pretratamiento, especialmente considerando el caso de la nitruración iónica que provee un gradiente de dureza que facilita la transición de la carga de la superficie al substrato. Los ensayos de indentación Rockwell C confirman este comportamiento, mostrando una mejor tenacidad a la fractura de los recubrimientos que fueron depositados sobre el acero nitrurado.

Estos resultados muestran que en una aplicación donde el desgaste predominante sea del tipo adhesivo, el recubrimiento va a actuar de forma correcta sin necesidad de utilizar un pretratamiento en el acero. Sin embargo, en el caso de un desgaste severo que involucre partículas duras, como es la abrasión o la erosión, la solicitación mecánica afectaría a la interface, y es ahí donde la nitruración ofrece un mayor soporte de carga, para evitar que el recubrimiento se delamine o se desprenda.

\section{Referencias}

Keunecke M., Stein C., Bewilogua K., Koelker W., Kassel D. y van den Berg H. (2010). Modified TiAlN coatings prepared by d.c. pulsed magnetron sputtering. Surface and Coatings Technology 205, 1273-1278. doi:10.1016/j.surfcoat.2010.09.023

Bouzakis, K. D., Michailidis, N., Skordaris, G., Bouzakis, E., Biermann, D. y M'Saoubi, R. (2012). Cutting with coated tools: Coating technologies, characterization methods and performance optimization. CIRP Annals - Manufacturing Technology 61, 2012, 703-723. doi:10.1016/j.cirp.2012.05.006

Erkens, G., Cremer, R., Hamoudi, T., Bouzakis, K. D., Mirisidis, I., Hadjiyiannis, S., Skordaris, G., Asimakopoulos, A., Kombogiannis, S., Anastopoulos, J. y Efstathiou, K. (2004). Properties and performance of high aluminum containing (Ti,Al)N based supernitride coatings in innovative cutting applications. Surface and Coatings Technology $177-178$, 727-734. doi:10.1016/j.surfcoat.2003.08.013

Dalibon, E. L., Heim, D., Forsich, C., Rosenkranz, A., Guitar, M. A. y Brühl, S. P. (2015). Characterization of thick and soft DLC coatings deposited on plasma nitrided austenitic stainless steel. Diamond \& Related Materials 59, 73-79. doi:10.1016/j.diamond.2015.09.010

Corengia, P., Ybarra G., Moina, C., Cabo A. y Broitman, E. (2005). Microstructural and topographical studies of DCpulsed plasma nitrided AISI 4140 low-alloy steel. Surface \& Coatings Technology 200, 2391-2397.

doi:10.1016/j.surfcoat.2005.01.060

Forsich, C., Dipolt, C., Heim, D., Mueller, T., Gebeshuber, A., Holecek, R. y Lugmair, C. (2014). Potential of thick a-C:H:Si films as substitute for chromium plating. Surface \& Coatings Technology 241, 86-92.

doi:10.1016/j.surfcoat.2013.11.011

Vidakis, N., Antoniandis, A. y Bilalis, N. (2003). The VDI 3198 indentation test evaluation of a reliable qualitative control for layered compounds. Journal of Materials Processing Technology 143, 481-485.

doi:10.1016/S0924-0136(03)00300-5 